\title{
Reptation of active entangled polymers SUPPLEMENTARY INFO
}

\author{
Andrés R. Tejedor and Jorge Ramírez* \\ Department of Chemical Engineering, \\ Universidad Politécnica de Madrid, \\ José Gutiérrez Abascal 2, 28006, Madrid, Spain
}

(Dated: August 2, 2019) 


\section{ANALYTICAL SOLUTION FOR THE TUBE SEGMENT SURVIVAL FUNC- TION.}

In order to compute the tube segment survival function $\psi(s, t)$, we start by calculating the function $\Psi(\xi, t ; s)$. The partial differential equation that describes the dynamics of $\Psi(\xi, t ; s)$ and the boundary and initial conditions are

$$
\begin{aligned}
& \frac{\partial \Psi}{\partial t}+c \frac{\partial \Psi}{\partial \tilde{\xi}}=D_{c} \frac{\partial^{2} \Psi}{\partial \tilde{\xi}^{2}} \\
& \Psi(\tilde{\xi}, 0 ; s)=\delta(\tilde{\xi}+s-L), \\
& \Psi(0, t ; s)=\Psi(L, t ; s)=0 .
\end{aligned}
$$

Following the procedure describe in [1], we can try solutions of the form

$$
\Psi(\tilde{\xi}, t ; s)=\phi(\tilde{\xi}, t ; s) \exp (\alpha \tilde{\xi}+\beta t) .
$$

where $\alpha$ and $\beta$ are arbitrary parameters. Substituting (2) in the differential equation (1), we get

$$
\frac{\partial \phi}{\partial t}+\left(\beta+c \alpha-D_{c} \alpha^{2}\right) \phi=\left(2 D_{c} \alpha-c\right) \frac{\partial \phi}{\partial \tilde{\xi}}+D_{c} \frac{\partial^{2} \phi}{\partial \tilde{\xi}}
$$

We can choose the values of $\alpha=c / 2 D_{c}$ and $\beta=-c^{2} / 4 D_{c}$ so that the differential equation (3) is simplified to

$$
\frac{\partial \phi}{\partial t}=D_{c} \frac{\partial^{2} \phi}{\partial \tilde{\xi}}
$$

with initial and boundary conditions given by

$$
\begin{aligned}
& \phi(\tilde{\xi}, 0 ; s)=\delta(\tilde{\xi}+s-L) \exp (-\alpha \tilde{\xi}), \\
& \phi(0, t ; s)=\phi(L, t ; s)=0 .
\end{aligned}
$$

which is a simple diffusion problem whose solution is well-known [2]:

$$
\phi(\tilde{\xi}, t ; s)=\sum_{p=1}^{\infty} a_{p} \exp \left(-\frac{p^{2} t}{\tau_{d}}\right) \sin \left(\frac{p \pi \tilde{\xi}}{L}\right),
$$

where $\tau_{d}$ is the terminal time defined in the main text. Enforcing the initial condition, the coefficients $a_{p}(0)$ are immediately obtained:

$$
\begin{aligned}
a_{p} & =\frac{2}{L} \int_{0}^{L} \delta(\tilde{\xi}+s-L) e^{-\alpha \tilde{\xi}} \sin \left(\frac{p \pi \tilde{\xi}}{L}\right) \\
& =\frac{2}{L} e^{-\alpha(L-s)} \sin \left(\frac{p \pi(L-s)}{L}\right) .
\end{aligned}
$$

\footnotetext{
* jorge.ramirez@upm.es
} 
Substitution of the coefficients $a_{p}$ into Eq. (6) yields

$$
\phi(\tilde{\xi}, t ; s)=\frac{2}{L} e^{-\alpha(L-s)} \sum_{p=1}^{\infty} \exp \left(\frac{-p^{2} t}{\tau_{d}}\right) \sin \left(\frac{p \pi(L-s)}{L}\right) \sin \left(\frac{p \pi \tilde{\xi}}{L}\right),
$$

Substituting back $\phi(\tilde{\xi}, t, a)$ into Eq. (2) and reverting the first change of variables, it is easy to show that the solution to the original problem (1) yields

$$
\Psi(\xi, t ; s)=\frac{2}{L} \exp \left(\frac{c \xi}{2 D_{c}}\right) \exp \left(\frac{-c^{2} t}{4 D_{c}}\right) \sum_{p=1}^{\infty} \exp \left(\frac{-p^{2} t}{\tau_{d}}\right) \sin \left(\frac{p \pi s}{L}\right) \sin \left(\frac{p \pi(s-\xi)}{L}\right) .
$$

\section{ANALYTICAL SOLUTION FOR THE MEAN SQUARE DISPLACEMENT.}

We shall first derive a stochastic equation for reptation with drift. Let $\Delta \xi(t)$ be the distance that the primitive chain moves in a time interval $\Delta t$. We can express $\mathbf{R}(s, t+\Delta t)=$ $\mathbf{R}(s+\Delta \xi(t), t)$ that relates the position of the tube segment $s$ at time $t+\Delta t$ to the position of the segment $s+\Delta \xi(t)$ at a previous time $t$. The distance $\Delta \xi(t)$ is a random Gaussian variable with mean and variance given by $\langle\Delta \xi(t)\rangle=c \Delta t,\left\langle\Delta \xi(t)^{2}\right\rangle=2 D_{c} \Delta t$. this distribution differs with pure reptation where the average of $\Delta \xi(t)$ is 0 . To compute the MSD we use the stochastic equation and the definition $g_{1}(s, t)=\phi(s, s ; t)=\left\langle(\mathbf{R}(s, t)-\mathbf{R}(s, 0))^{2}\right\rangle$. It is

easier to compute the more general correlation function $\phi\left(s, s^{\prime} ; t\right)=\left\langle\left(\mathbf{R}(s, t)-\mathbf{R}\left(s^{\prime}, 0\right)\right)^{2}\right\rangle$. Expanding stochastic equation we get the partial differential equation

$$
\frac{\partial \phi\left(s, s^{\prime} ; t\right)}{\partial t}-c \frac{\partial \phi\left(s, s^{\prime} ; t\right)}{\partial s}=D_{c} \frac{\partial^{2} \phi\left(s, s^{\prime} ; t\right)}{\partial s^{2}} .
$$

The boundary and initial conditions are preserved from the pure reptation problem:

$$
\phi\left(s, s^{\prime} ; 0\right)=a\left|s-s^{\prime}\right|, \quad\left\{\begin{array}{l}
\left.\frac{\partial \phi\left(s, s^{\prime} ; t\right)}{\partial s}\right|_{s=L}=a \\
\left.\frac{\partial \phi\left(s, s^{\prime} ; t\right)}{\partial s}\right|_{s=0}=-a
\end{array}\right.
$$

In order to turn the boundary conditions homogeneous, we start splitting $\phi\left(s, s^{\prime} ; t\right)$ into two different functions:

$$
\phi\left(s, s^{\prime} ; t\right)=\psi\left(s, s^{\prime} ; t\right)+\rho\left(s, s^{\prime}\right)
$$

where $\rho\left(s, s^{\prime}\right)=a\left|s-s^{\prime}\right|$. Substituting (12) into the original problem, the equation to solve for $\psi\left(s, s^{\prime} ; t\right)$ is written as

$$
\frac{\partial \psi}{\partial t}-c \frac{\partial \psi}{\partial s}-D_{c} \frac{\partial^{2} \psi}{\partial s^{2}}=F\left(s, s^{\prime}\right)
$$


with homogeneous both initial and boundary conditions, i.e.

$$
\left.\psi\left(s, s^{\prime} ; t\right)\right|_{t=0}=\left.0 \quad \frac{\partial \psi}{\partial s}\right|_{s=0}=\left.\frac{\partial \psi}{\partial s}\right|_{s=L}=0
$$

and where

$$
F\left(s, s^{\prime}\right)=a c\left[\Theta\left(s-s^{\prime}\right)-1\right]+2 a D_{c} \delta\left(s-s^{\prime}\right),
$$

being $\Theta\left(s-s^{\prime}\right)$ the Heaviside function and $\delta\left(s-s^{\prime}\right)$ the Dirac delta. Note that the nonhomogeneous term $F\left(s, s^{\prime}\right)$ arises from the substitution of $\rho\left(s, s^{\prime}\right)$ in Eq. (10). The function $\psi\left(s, s^{\prime} ; t\right)$ can be factorized as follows

$$
\psi\left(s, s^{\prime} ; t\right)=f(s) \cdot T\left(s^{\prime}, t\right)
$$

For the homogeneous problem, the segmental equation for $f(s)$ can be expressed as

$$
\frac{\partial^{2} f}{\partial s^{2}}+2 d \frac{\partial f}{\partial s}+\lambda_{p}^{2} f=0
$$

where $\lambda_{p}$ are the eigenvalues and

$$
d=\frac{c}{2 D_{c}}
$$

The boundary conditions for $f(s)$ are given in Eq. (14). The solution to this problem with the homogeneous boundary conditions is the following [3]:

$$
\begin{array}{ll}
f_{p}(s)=e^{-d s}\left[\cos \left(\frac{p \pi s}{L}\right)+\frac{d L}{p \pi} \sin \left(\frac{p \pi s}{L}\right)\right] & \text { if } \quad p \in \mathbb{N} \\
f_{0}(s)=1 & \text { if } \quad \lambda_{0}^{2}=0 .
\end{array}
$$

Note that the eigenvalues in the first case take the form

$$
\lambda_{p}^{2}=d^{2}+\left(\frac{p \pi}{L}\right)^{2}
$$

We shall now expand $F\left(s, s^{\prime}\right)$ in the basis of the eigenfunctions (19):

$$
\begin{aligned}
2 a D_{c} \delta\left(s-s^{\prime}\right) & =\sum_{p=0}^{\infty} B_{p}\left(s^{\prime}\right) f_{p}(s), \\
a c\left[2 \Theta\left(s-s^{\prime}\right)-1\right] & =\sum_{p=0}^{\infty} C_{p}\left(s^{\prime}\right) f_{p}(s) .
\end{aligned}
$$


The coefficient of the series can be computed by multiplying the sum by $\exp (2 d s) f_{q}(s)$ for $p>0$ and by $\exp (2 d s)$ for $p=0$ and integrating between 0 and $L$ :

$$
\begin{aligned}
& \left\{\begin{array}{l}
B_{0}\left(s^{\prime}\right)=4 a d D_{c} \frac{\exp \left(2 d s^{\prime}\right)}{\exp (2 d L)-1} \\
B_{p}\left(s^{\prime}\right)=\frac{4 a D_{c} p^{2} \pi^{2} \exp \left(2 d s^{\prime}\right)}{L\left(L^{2} d^{2}+p^{2} \pi^{2}\right)} f_{p}\left(s^{\prime}\right) \quad p \in \mathbb{N},
\end{array}\right. \\
& \left\{\begin{array}{l}
C_{0}\left(s^{\prime}\right)=a c \frac{2 \exp \left(2 d s^{\prime}\right)-\exp (2 d L)-1}{1-\exp (2 d L)} \\
C_{p}\left(s^{\prime}\right)=-4 a c p \pi \frac{\exp \left(d s^{\prime}\right) \sin \left(\frac{p \pi s^{\prime}}{L}\right)}{p^{2} \pi^{2}+L^{2} d^{2}} \quad p \in \mathbb{N} .
\end{array}\right.
\end{aligned}
$$

Efforts must now be focused on solving the temporal equation which is written as

$$
\frac{\partial T}{\partial t}+D_{c} \lambda_{p}^{2} T=F_{p}\left(s, s^{\prime}\right)
$$

where $F_{p}\left(s, s^{\prime}\right)$ corresponds to the expansion in terms of the spatial eigenfunctions as described above. For the zero mode Eq. (23) is simplified to

$$
\frac{\partial T}{\partial t}=B_{0}\left(s^{\prime}\right)+C_{0}\left(s^{\prime}\right)
$$

which can be straightforwardly integrated to give in a simplified form

$$
T_{0}(t)=a c \operatorname{coth}(d L) t=\psi_{0}\left(s, s^{\prime} ; t\right)
$$

The latter relation is valid since $f_{0}(s)=1$. Equivalently proceeding for the $p>0$ modes, the temporal equation (23) with the eigenvalues (20) yields

$$
T_{p}(t)=D_{p}(0) \exp \left(-D_{c} \lambda_{p}^{2} t\right)+\frac{F_{p}\left(s, s^{\prime}\right)}{\lambda_{p}^{2}} .
$$

The first term corresponds to the solution for the homogeneous equation with $D_{p}(0)$ to be estimated by enforcing the initial condition (14), so that

$$
D_{p}(0)=-\frac{F_{p}\left(s, s^{\prime}\right)}{\lambda_{p}^{2}},
$$

and hence, substituting the full expansion of $F_{p}\left(s, s^{\prime}\right)$ of the positive modes we get

$$
\begin{aligned}
\psi_{p}\left(s, s^{\prime} ; t\right) & =e^{d\left(s^{\prime}-s\right)} \sum_{p=1}^{\infty}\left(1-e^{-D_{c} \lambda_{p}^{2} t}\right) \frac{4 a L p^{2} \pi^{2}}{\left(p^{2} \pi^{2}+L^{2} d^{2}\right)^{2}} \\
\times & {\left[\cos \left(\frac{p \pi s^{\prime}}{L}\right)-\frac{d L}{p \pi} \sin \left(\frac{p \pi s^{\prime}}{L}\right)\right]\left[\cos \left(\frac{p \pi s}{L}\right)+\frac{d L}{p \pi} \sin \left(\frac{p \pi s}{L}\right)\right], }
\end{aligned}
$$


Finally, putting together both eigenfunctions (Eqs. (25), (28)) and adding the function $\rho\left(s, s^{\prime}\right)$, we get the full solution for the segmental motion:

$$
\begin{aligned}
\phi\left(s, s^{\prime} ; t\right)= & a\left|s-s^{\prime}\right|+\operatorname{act} \operatorname{coth}\left(\frac{c L}{2 D_{c}}\right)+e^{\left(\frac{c\left(s^{\prime}-s\right)}{2 D_{c}}\right)} \sum_{p=1}^{\infty}\left(1-e^{-D_{c} \lambda_{p}^{2} t}\right) \frac{64 a D_{c}^{4} L p^{2} \pi^{2}}{\left(4 D_{c}^{2} p^{2} \pi^{2}+L^{2} c^{2}\right)^{2}} \\
& \times\left[\cos \left(\frac{p \pi s^{\prime}}{L}\right)-\frac{c L}{2 D_{c} p \pi} \sin \left(\frac{p \pi s^{\prime}}{L}\right)\right]\left[\cos \left(\frac{p \pi s}{L}\right)+\frac{c L}{2 D_{c} p \pi} \sin \left(\frac{p \pi s}{L}\right)\right] .
\end{aligned}
$$

[1] A. Mojtabi and M. O. Deville, One-dimensional linear advection-diffusion equation: Analytical and finite element solutions, Computers \& Fluids 107, 189 (2015).

[2] M. Doi and S. Edwards, The Theory of Polymer Dynamics, Vol. 73 (Oxford University Press, 1988).

[3] G. F. Simmons, Differential equations with applications and historical notes (CRC Press, 2016). 